\title{
Trajetória de Acumulação de Competências Tecnológicas e Processos de Aprendizagem: proposta de modelo analítico para agências de viagens e operadoras turísticas
}

\author{
Trajectory of the Accumulation of Technological Capability and Learning Processes: proposed \\ analytical model for travel agencies and tour operators
}

Recebido em 02.10.2013. Aprovado em 03.07. 2013

Avaliado pelo sistema double blind review

\begin{abstract}
Saulo Barroso Rocha
saulorocha@empreendedorismo.uff.br

Programa de Pós-graduação em Gestão e Empreendedorismo da Universidade Federal Fluminense - Niterói RJ - Brasil
\end{abstract}

\section{Deborah Moraes Zouain}

deborah.zouain@fgv.br

Escola Brasileira de Administração Pública e de Empresas-EBAPE da Fundação Getulio Vargas/FGV - Rio de Janeiro - RJ - Brasil

\section{Resumo}

Este artigo tem como objetivo principal propor modelo de identificação de trajetórias de acumulação de competências tecnológicas para empresas do setor de agenciamento de viagens e operações turísticas, e examinar o impacto dos processos de aprendizagem sobre estas trajetórias. Para atingir tal objetivo, buscou-se a literatura de inovação e aprendizagem tecnológica em organizações de países industrializados e em processo de industrialização. A maioria dos estudos que descrevem modelos de avaliação da capacidade inovadora e de aprendizagem tecnológica focaliza indústrias. Nesta literatura, escassas são as pesquisas que tratam do setor deagenciamento de viagens. A partir desta teoria, foram adaptadas duas estruturas para as empresas do setor de agências de viagens: a matriz de competências tecnológicas e a de análise de processos de aprendizagem. Com base nas evidências na literatura, verificou-se que: (1) o modelo proposto pode ser adequado para identificar a trajetória da acumulação de competências tecnológicas neste setor; (2) a combinação de diferentes mecanismos de aprendizagem pode impulsionar a evolução desta trajetória; e (3) além desta variedade de ações, a recorrência, o funcionamento e a interação entre os diferentes processos de aprendizagem potencializam a conversão do conhecimento do nível individual para sistemas e rotinas organizacionais, possibilitando um maior nível de competitividade para estas organizações. Propõe-se uma generalização analítica da metodologia do estudo para outras empresas do mesmo setor, a fim de ampliar as evidências de agências e operadoras, e contribuir para a prática de gestão destas organizações.

Palavras-chave: Inovação, Competências Tecnológicas, Processos de Aprendizagem, Turismo, Agências de Viagens.

\section{Abstract}

The main goal of this article is to propose a model identifying trajectories of the accumulation of technological capabilities for companies in the travel agency and tour operations industry, and examine the impact of learning processes on these trajectories. To achieve this goal, we looked for the literature in technological learning and innovation organizations in industrialized countries and industrialization process. Most studies that describe models for evaluating innovative capacity and technological learning focus on industries. In those literature is very rare to find anything that adresses the sector of travel agencies. From this theory, two structures were 
adapted to the travel agencies industry: a matrix of skills and technological analysis and of learning processes. Based on the evidences in the literature, it was found that : ( 1 ) the proposed model may be appropriate to identify the trajectory of technological capability accumulation in this sector , $(2)$ the combination of different learning mechanisms can drive the evolution of this trend ; and ( 3 ) beyond this range of actions, recurrence , operation and interaction between the different learning processes potencialize the knowledge conversion of individual systems and organizational routines, enabling a higher level of competitiveness for these organizations. We propose a generalization of the analytical methodology of the study to other companies in the same industry in order to expand the evidence from agencies and operators, and contribute to the practice of management of these organizations.

keyword: Innovation, Technological Capability, and Learning Processes, Tourism, Travel Agency.

\section{Introdução}

Este artigo propõe modelo analítico para identificar trajetórias de acumulação de competências tecnológicas e examinar o impacto dos processos de aprendizagem para estas trajetórias, por meio da aplicação de estruturas analíticas em empresas do setor de agenciamento e operações de viagens. Competência tecnológica nesta pesquisa é definida como sendo os recursos necessários para gerar e conduzir melhorias em processos e produtos (Bell \& Pavitt, 1993, 1995).

Estudos na literatura de empresas em países desenvolvidos relativos à acumulação de competências tecnológicas (Bell \& Pavitt,, 1993; Kim, 1997, 1999; Dutrénit, 2000, 2004; Figueiredo, 2001, 2009; Lam, 2005) sugerem que os processos de aprendizagem influenciam a acumulação destas competências nas organizações, sendo esta influência tanto positiva quanto negativa. Nos países em desenvolvimento, as empresas em industrialização são organizações que atuam em economias 'em desenvolvimento', ou de industrialização recente. Estas empresas diferem das localizadas em economias desenvolvidas e com base nestas diferenças foram desenvolvidas estruturas analíticas para identificar trajetórias de competências tecnológicas (Ariffin \& Bell, 1999; Ariffin \& Figueiredo, 2004; Bell, 1984; Bell \& Giuliani, 2007; Dutrénit, 2000, 2004; Figueiredo, 2001, 2002a, 2009; Lall, 1992).

A identificação da trajetória da acumulação de competências tecnológicas, nas empresas de agenciamento e operação de viagens, contribui para a prática de gestores dessas empresas - acentuando ou revertendo modelos estabelecidos de processos de aprendizagem. Além disso, busca explicar até que ponto os estudos desenvolvidos na literatura de empresas em industrialização, pertinentes aos estudos sobre acumulação de competências tecnológicas (Lall, 1992; Pattel \& Pavitt, 1994; Bell \& Pavitt, 1995; Souitaris, 2002; Figueiredo, 2003; De Jong e Marsili, 2006; Castellacci, 2008; Cavalcanti \& Figueiredo, 2006) -, permitem identificar a trajetória da acumulação de competências tecnológicas no setor de agenciamento de viagens e operação turística e entender como os processos subjacentes de aprendizagem influenciam esta trajetória.

O cenário concorrente atual do setor de Turismo é fruto de transformações observadas, notadamente, nas últimas três décadas em que se destacam as inovações tecnológicas e seus impactos na economia e na administração empresarial. Especificamente, no setor de agenciamento e operações de viagens, inovações em processos (p.ex.: novas tecnologias de informação e comunicação) e produtos (p. ex.: viagens de baixo custo, novos segmentos de mercado) alteraram as relações de consumo neste setor, como por exemplo a possibilidade da interação direta entre clientes e meios de hospedagem. O setor de turismo na economia brasileira tem se destacado como importante impulsionador de desenvolvimento socioeconômico para o país (Brasil, 2011). Para as empresas deste setor, após a tomada dos recursos técnicos e financeiros advindos dos 


\section{Trajetória de Acumulação de Competências Tecnológicas e Processos de Aprendizagem: proposta de modelo analítico para agências de viagens e operadoras turísticas}

programas federais, o desafio passa a ser como acumular competências tecnológicas com, conseqüente, incremento da sua competitividade nos mercados nacional e internacional.

\section{Inovação e Acumulação de Competências e Tecnológicas}

Em termos mais gerais, agentes privados que buscam o lucro alocarão recursos para a exploração e desenvolvimento de novos produtos e novas técnicas, se eles sabem ou acreditam na existência de algum tipo de oportunidade técnica ou científica ainda não explorada; na existência de um mercado para seus novos produtos e serviços e, finalmente, se existir a possibilidade de benefício econômico (Dosi, 1988a, 1988b; Lazonick, 2005). Dosi (1988a) argumenta que se observamos a maior parte das soluções que resultam em inovações tecnológicas, constatamos a utilização de uma variedade de conhecimentos.

Essas afirmações estão alinhadas com o resultado de estudos que identificam uma quantidade significativa de inovações e aperfeiçoamentos originada pelo "aprender fazendo" e pelo "aprender usando" (Arrow, 1962; Rosenberg, 1982; Teece, 1977, 1986; Pavitt, 1984; Figueiredo, 2002a, 2004). Para Pavitt (2005), uma corporação constrói sobre seu conhecimento acumulado de produtos, processos e práticas organizacionais. Esta mesma afirmação é também ratificada por Cohen e Levinthal $(1989,1990)$, que argumentam que os produtos da inovação dependem da acumulação prévia de conhecimento.

Esta característica da 'cumulatividade' pode passar despercebida na maioria das organizações, pois são incrementais e não possuem a visibilidade de "grandes inovações" de alto impacto mercadológico. Porém, inovações incrementais, no contexto do processo cumulativo contínuo, envolvem uma série de estratégias e práticas de gestão que pode trazer benefícios, como: custos menores de produção do que os concorrentes, novas rotinas e procedimentos organizacionais, novas características aos produtos e serviços existentes (Dodgson et al, 2008).

Dosi (1988) e Dosi, Marengo e Pasquali (2006) afirmam que fatores importantes para a busca por inovações estão relacionados com as características do conhecimento e da solução de problemas. A heurística sobre "como fazer as coisas" e "como melhorá-las" estão muitas vezes incorporadas às rotinas organizacionais, que fazem certas empresas "boas" para explorar oportunidades técnicas e traduzi-las em produtos comercializáveis. Em tais questões, há uma quantidade significativa de indivisibilidade organizacional.

Por conta desta necessidade de acumulação de conhecimento, tácito ou explícito, uma implicação crucial é que as atividades inovadoras são fortemente seletivas, cumulativas na aquisição de capacidades de solução de problemas e definem, invariavelmente, uma trajetória tecnológica (Dosi, 1988a). Uma mudança de paradigma ( $p$. ex.: 0 advento das agências de viagens no ambiente Internet, ou agências de viagens on-line) em determinado setor, geralmente implica uma mudança na trajetória: juntamente com as diferentes bases de conhecimento que trazem mudanças para as dimensões da inovação. De acordo com Teece (1986), durante uma mudança de paradigma em determinado setor, as empresas buscam melhorar e diversificar sua tecnologia, procurando zonas que lhes permitam utilizar a sua base tecnológica (produtos e processos) e construir novas competências sobre essa mesma base e acumular novas informações sobre seus mercados existentes. Se essas zonas podem ser identificadas, medidas ou explicadas, também é possível prever os prováveis padrões de atividades inovadoras nas empresas, indústrias e países (Pavitt, 1984; Dosi, Pavitt \& Soete, 1992). 
Em geral, a formação da trajetória tecnológica incorpora a incerteza associada às atividades inovadoras, que envolve não somente a falta de conhecimento preciso sobre custos e resultados, como também a deficiência de conhecimento sobre as alternativas possíveis de resultados futuros (Freeman, 1982; Bruland \& Mowery, 2005).

O processo de construção de competências tecnológicas tem sido um tema de atenção na literatura sobre as empresas industriais nos últimos 20 anos (Dutrénit, 2004).

Como competência tecnológica, entende-se os recursos necessários para gerar e administrar melhorias em investimentos, processos e organização da produção, produtos e equipamentos. Esses recursos são acumulados e incorporados em indivíduos (experiência e habilidades, conhecimento tácito) e sistemas organizacionais (Bell \& Pavitt, 1993, 1995). Kim (1997) afirma que, nessa literatura, recursos tecnológicos são entendidos como a capacidade de utilizar os conhecimentos tecnológicos de forma eficiente para assimilar, utilizar, adaptar e mudar as tecnologias existentes, e também como a capacidade de criar novas tecnologias e desenvolver novos produtos e processos. Esta definição está inter-relacionada com as desenvolvidas anteriormente por Katz (1984, 1987), Lall $(1987,1992)$, Bell $(1984,2006)$ e Dahlman, C., Ross-larsen, B., Westphal (1987).

Segundo Lall (1992), existem várias formas de categorizar os níveis de competências ou capacidades tecnológicas de uma empresa. E baseado em Katz (1984, 1987), Dahlman, Ross-Larson e Westphal (1987) e Lall (1987), é construída a matriz de competências. Em Lall (1992), esta matriz apresenta uma taxonomia de capacidade tecnológica por funções técnicas.

Bell e Pavitt (1993) definem as principais características chave para acumulação de competências tecnológicas em países industrializados, que podem servir como base para estudos em países em industrialização. Estas características são: recursos iniciais, conhecimento tácito e específico, a importância central das empresas, as conexões entre empresas, o processo de acumulação, indústrias como criadoras de capital humano, e a complementaridade entre importar tecnologia e acumulação local de tecnologia. Ainda segundo os autores, os padrões e trajetórias da acumulação tecnológica diferem entre firmas e indústrias; como consequências, observa-se que países desenvolvidos diferem, de forma marcante, em suas trajetórias de acumulação tecnológica. Essas diferenças na trajetória são confirmadas no Brasil pelos estudos de Figueiredo $(2003,2004)$.

\section{Estrutura Analítica - Matriz de Competências Tecnológicas}

A partir do momento que a empresa em industrialização inicia a operação sob condições não competitivas no mercado externo, o problema básico da 'maturação empresarial' é a acumulação de competências tecnológicas para se tornar e permanecer no cenário externo como uma empresa capaz de oferecer produtos e serviços competitivos (Bell, Ross-Larson \& Westphal, 1984). Essa acumulação parece envolver uma sequência de esforços tecnológicos internos (Katz, 1984, 2001). Por este motivo, é possível iniciar com a mais básica competência tecnológica e, com base nela, evoluir para outras competências de mais alto nível de desenvolvimento tecnológico (Figueiredo, 2003, 2004, 2009). Figueiredo et al (2010) distingue sete níveis de acumulação de competências, e Dutrénit e Vera-Cruz (2005) introduzem funções técnicas relacionadas com as conexões internas dentro das empresas.

A estrutura descrita na Tabela 1 foi adaptada de Figueiredo (2010) e tem como objetivo mensurar os tipos e níveis de competências tecnológicas. A estrutura indicada na Tabela 1 sofreu as adaptações de conteúdo em comparação com as estruturas construídas na literatura recente. Essas adaptações referem-se às características de competências específicas para o setor de agenciamento de viagens e operações turísticas. Em uma primeira fase, estas adaptações foram discutidas em reunião ocorrida em novembro de 2010 na Associação Brasileira de Agentes de Viagens (ABAV). O segundo momento foi dedicado a realização de três 


\section{Trajetória de Acumulação de Competências Tecnológicas e Processos de Aprendizagem: proposta de modelo analítico para agências de viagens e operadoras turísticas}

grupos focais com as empresas do setor de receptivo, operadores e agências de viagens em geral, cujo objetivo foi discutir e definir as competências tecnológicas caracterísitcas do setor, bem como os variados processos de aprendizagem utilizados na capacitação de funcionários. Finalmente, neste processo de construção das matrizes, em 2011, aplicaram-se os intrumentos (matriz de competências e de processos de aprendizagem) em duas agências de viagens na cidade do Rio de Janeiro com o objetivo de refinar a proposta de modelo.. A estrutura, em forma de matriz, identifica quatro funções tecnológicas: Produtos e Serviços, Gestão Financeira, Planejamento e Gestão, Promoção e Vendas. Estas funções foram consideradas fundamentais para a gestão de agências de viagens e operadores turísticos na identificação de diferentes tipos e níveis de inovação. Esta separação das funções tecnológicas alinha-se aos autores Patel e Pavitt (1997), pois segundo eles as competências tecnológicas específicas de determinada firma ajudam a explicar como as empresas mudam através do tempo, e se são capazes ou não de se manterem competitivas.

Para atingir determinados níveis de competências tecnológicas, são requeridas amplas capacidades técnicas e administrativas que somente podem ser adquiridas por um longo processo de aprendizagem (Karaoz \& Albeni, 2005).

TABELA 1 - Competências tecnológicas para o setor de agenciamento de viagens e operações turísticas

\begin{tabular}{|c|c|c|c|c|}
\hline \multirow{2}{*}{$\begin{array}{c}\text { NÍVEL DE } \\
\text { ACUMULAÇÃO } \\
\text { DE } \\
\text { COMPETÊNCIAS } \\
\text { TECNOLÓGICAS }\end{array}$} & \multicolumn{4}{|c|}{$\begin{array}{c}\text { MATRIZ DE COMPETÊNCIAS TECNOLÓGICAS PARA O SETOR DE AGENCIAMENTO } \\
\text { DE VIAGENS E OPERAÇÕES TURÍSTICAS }\end{array}$} \\
\hline & $\begin{array}{l}\text { PRODUTOS E } \\
\text { SERVIÇOS }\end{array}$ & $\begin{array}{c}\text { GESTÃO } \\
\text { FINANCEIRA E } \\
\text { DE } \\
\text { INVESTIMENTOS }\end{array}$ & $\begin{array}{l}\text { PLANEJAMENTO E } \\
\text { GESTÃO } \\
\text { (Planejamento, } \\
\text { Qualidade } \\
\text { Atendimento, } \\
\text { Recursos Humanos, } \\
\text { Tecnologia da } \\
\text { Informação) }\end{array}$ & $\begin{array}{l}\text { PROMOÇÃO E } \\
\text { VENDAS }\end{array}$ \\
\hline
\end{tabular}




\begin{tabular}{|c|c|c|c|c|}
\hline \multirow{2}{*}{\begin{tabular}{|c} 
NÍVEL DE \\
ACUMULAÇÃO \\
DE \\
COMPETÊNCIAS \\
TECNOLÓGICAS
\end{tabular}} & \multicolumn{4}{|c|}{$\begin{array}{c}\text { MATRIZ DE COMPETÊNCIAS TECNOLÓGICAS PARA O SETOR DE AGENCIAMENTO } \\
\text { DE VIAGENS E OPERAÇÕES TURÍSTICAS }\end{array}$} \\
\hline & $\begin{array}{l}\text { PRODUTOS E } \\
\text { SERVIÇOS }\end{array}$ & $\begin{array}{c}\text { GESTÃO } \\
\text { FINANCEIRA E } \\
\text { DE } \\
\text { INVESTIMENTOS }\end{array}$ & $\begin{array}{l}\text { PLANEJAMENTO E } \\
\text { GESTÃO } \\
\text { (Planejamento, } \\
\text { Qualidade } \\
\text { Atendimento, } \\
\text { Recursos Humanos, } \\
\text { Tecnologia da } \\
\text { Informação) }\end{array}$ & $\begin{array}{l}\text { PROMOÇÃO E } \\
\text { VENDAS }\end{array}$ \\
\hline Básico & $\begin{array}{l}\text { Possui conhecimento } \\
\text { básico dos produtos } \\
\text { turísticos } \\
\text { comercializados. } \\
\text { Estabelece parceria com } \\
\text { consolidadoras para a } \\
\text { emissão de passagens. } \\
\text { Assessora o cliente nas } \\
\text { demandas da viagem } \\
\text { (p.ex.: normas de } \\
\text { embarque, passaporte } \\
\text { etc.). } \\
\text { Oferece atendimento em } \\
\text { idioma estrangeiro com } \\
\text { domínio básico da língua. } \\
\text { Entende e acompanha as } \\
\text { mudanças na legislação } \\
\text { geral do Turismo. }\end{array}$ & $\begin{array}{l}\text { Realiza } \\
\text { monitoramento } \\
\text { diário dos valores } \\
\text { de entrada e saída } \\
\text { de recursos. } \\
\text { Utiliza fluxo de } \\
\text { caixa como } \\
\text { ferramenta de } \\
\text { controle } \\
\text { financeiro. Os } \\
\text { recursos para } \\
\text { investimento são } \\
\text { provenientes de } \\
\text { capital próprio. } \\
\text { Fonte de receita } \\
\text { principal } \\
\text { proveniente da } \\
\text { venda de produtos } \\
\text { turísticos. }\end{array}$ & $\begin{array}{l}\text { Identificam de maneira } \\
\text { não estruturada as } \\
\text { tendências de mercado. } \\
\text { Possui conhecimento } \\
\text { incipiente sobre o } \\
\text { ambiente de negócios } \\
\text { no qual opera. } \\
\text { Estabelece e divulga } \\
\text { missão e visão da } \\
\text { empresa. } \\
\text { Trabalha com guias } \\
\text { credenciados pelos } \\
\text { órgãos competentes. } \\
\text { Monitora de forma não } \\
\text { estruturada as } \\
\text { demandas dos clientes. } \\
\text { Pratica o pagamento de } \\
\text { comissão por venda aos } \\
\text { funcionários. } \\
\text { Utiliza programas } \\
\text { básicos de computador } \\
\text { como editores de texto } \\
\text { e de planilha. Correio } \\
\text { eletrônico é a forma de } \\
\text { contato usual com } \\
\text { parceiros e } \\
\text { fornecedores. } \\
\text { Estabelece contrato de } \\
\text { utilização de portal de } \\
\text { reservas das Cias. } \\
\text { aéreas e de outros } \\
\text { fornecedores. }\end{array}$ & $\begin{array}{l}\text { Considera o } \\
\text { contato pessoal } \\
\text { (rede de } \\
\text { relacionamentos) } \\
\text { como a base da } \\
\text { divulgação e } \\
\text { promoção. } \\
\text { Possui material } \\
\text { impresso } \\
\text { somente em } \\
\text { português. }\end{array}$ \\
\hline & & & & h-Abr 2013 * 64-81 \\
\hline
\end{tabular}




\begin{tabular}{|c|c|c|c|c|}
\hline \multirow{2}{*}{$\begin{array}{c}\text { NÍVEL DE } \\
\text { ACUMULAÇÃO } \\
\text { DE } \\
\text { COMPETÊNCIAS } \\
\text { TECNOLÓGICAS }\end{array}$} & \multicolumn{4}{|c|}{$\begin{array}{c}\text { MATRIZ DE COMPETÊNCIAS TECNOLÓGICAS PARA O SETOR DE AGENCIAMENTO } \\
\text { DE VIAGENS E OPERAÇÕES TURISTICAS }\end{array}$} \\
\hline & $\begin{array}{l}\text { PRODUTOS E } \\
\text { SERVIÇOS }\end{array}$ & $\begin{array}{c}\text { GESTÃO } \\
\text { FINANCEIRA E } \\
\text { DE } \\
\text { INVESTIMENTOS }\end{array}$ & $\begin{array}{l}\text { PLANEJAMENTO E } \\
\text { GESTÃO } \\
\text { (Planejamento, } \\
\text { Qualidade } \\
\text { Atendimento, } \\
\text { Recursos Humanos, } \\
\text { Tecnologia da } \\
\text { Informação) }\end{array}$ & $\begin{array}{l}\text { PROMOÇÃO E } \\
\text { VENDAS }\end{array}$ \\
\hline $\begin{array}{c}(2) \\
\text { Intermediário }\end{array}$ & $\begin{array}{l}\text { Possui conhecimento } \\
\text { intermediário, que vai } \\
\text { além das informações } \\
\text { elementares do produto } \\
\text { turístico comercializado. } \\
\text { Analisa melhorias em } \\
\text { produtos e serviços, a } \\
\text { partir de problemas } \\
\text { informados pelos clientes. } \\
\text { Comercializa } \\
\text { manifestações e } \\
\text { produções culturais como } \\
\text { produtos turísticos ou } \\
\text { valor agregado aos } \\
\text { destinos (p. ex.: São João } \\
\text { em Caruaru, Festa do } \\
\text { Círio de Nazareth em } \\
\text { Belém etc.). }\end{array}$ & $\begin{array}{l}\text { Realiza análise } \\
\text { parcial dos } \\
\text { processos } \\
\text { financeiros da } \\
\text { empresa. } \\
\text { Possui núcleo } \\
\text { responsável pela } \\
\text { gestão financeira. }\end{array}$ & 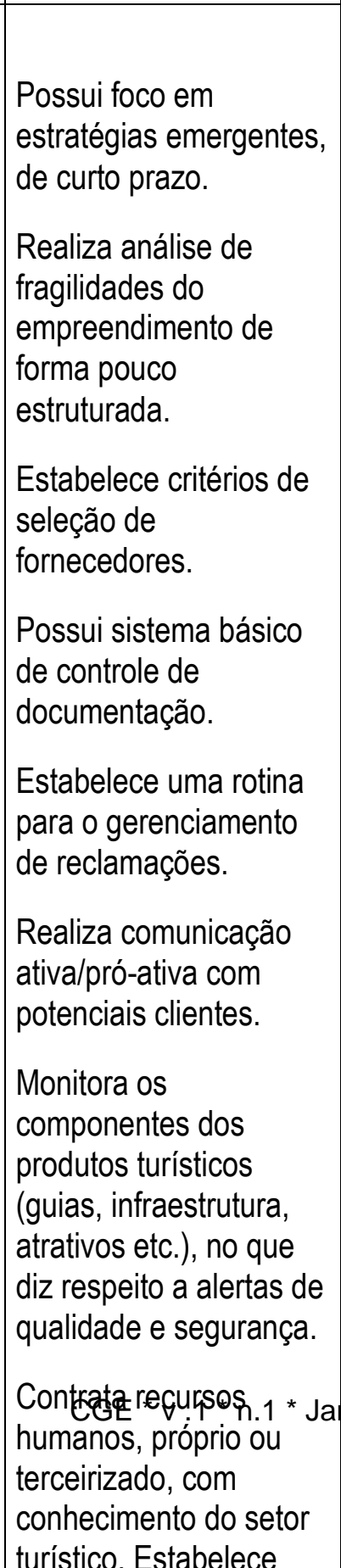 & $\begin{array}{l}\text { Participa como } \\
\text { visitante em feiras } \\
\text { e eventos } \\
\text { nacionais de } \\
\text { Turismo. } \\
\text { Utiliza } \\
\text { ferramentas como } \\
\text { correio eletrônico } \\
\text { e portais de } \\
\text { Turismo para } \\
\text { promoção e } \\
\text { divulgação. } \\
\text { Possui material } \\
\text { promocional } \\
\text { impresso em } \\
\text { mais de um } \\
\text { idioma. }\end{array}$ \\
\hline
\end{tabular}




\begin{tabular}{|c|c|c|c|c|}
\hline \multirow{2}{*}{\begin{tabular}{|c} 
NIIVEL DE \\
ACUMULAÇÃO \\
DE \\
COMPETÊNCIAS \\
TECNOLÓGICAS
\end{tabular}} & \multicolumn{4}{|c|}{$\begin{array}{c}\text { MATRIZ DE COMPETÊNCIAS TECNOLÓGICAS PARA O SETOR DE AGENCIAMENTO } \\
\text { DE VIAGENS E OPERAÇÕES TURÍSTICAS }\end{array}$} \\
\hline & $\begin{array}{l}\text { PRODUTOS E } \\
\text { SERVIÇOS }\end{array}$ & $\begin{array}{c}\text { GESTÃO } \\
\text { FINANCEIRA E } \\
\text { DE } \\
\text { INVESTIMENTOS }\end{array}$ & $\begin{array}{l}\text { PLANEJAMENTO E } \\
\text { GESTÃO } \\
\text { (Planejamento, } \\
\text { Qualidade } \\
\text { Atendimento, } \\
\text { Recursos Humanos, } \\
\text { Tecnologia da } \\
\text { Informação) }\end{array}$ & $\begin{array}{l}\text { PROMOÇÃO E } \\
\text { VENDAS }\end{array}$ \\
\hline $\begin{array}{c}\text { (3) } \\
\text { Intermediário } \\
\text { Superior }\end{array}$ & $\begin{array}{l}\text { Realiza visitas técnicas e } \\
\text { conhece detalhadamente, } \\
\text { de modo avançado, os } \\
\text { produtos comercializados. } \\
\text { Comercializa produtos } \\
\text { e/ou serviços turísticos } \\
\text { com parceiros } \\
\text { internacionais. } \\
\text { Possui habilidade e } \\
\text { fluência em diferentes } \\
\text { idiomas ( } \geq 3 \text { ). } \\
\text { Elabora ou comercializa } \\
\text { pacotes turísticos } \\
\text { especializados para } \\
\text { determinados segmentos } \\
\text { de mercado. }\end{array}$ & $\begin{array}{l}\text { É capaz de } \\
\text { executar rotina de } \\
\text { planejamento } \\
\text { financeiro. } \\
\text { Forma preço com } \\
\text { base no mercado. } \\
\text { É capaz de } \\
\text { calcular e } \\
\text { acompanhar ponto } \\
\text { de equilíbrio do } \\
\text { negócio. } \\
\text { Possui fonte de } \\
\text { receita mensal } \\
\text { proveniente da } \\
\text { venda de produtos } \\
\text { turísticos e } \\
\text { produção } \\
\text { associada. }\end{array}$ & $\begin{array}{l}\text { Estabelece formalmente } \\
\text { plano estratégico para } \\
\text { curto e médio prazos, e } \\
\text { divulga para toda } \\
\text { empresa. } \\
\text { Adota práticas internas } \\
\text { de redução de gastos } \\
\text { com energia, material } \\
\text { de escritório etc. } \\
\text { Estabelece parceria } \\
\text { com fornecedores } \\
\text { internacionais de } \\
\text { produtos e/ou serviços } \\
\text { turísticos. } \\
\text { Integra arranjo } \\
\text { associativo nacional. } \\
\text { Possui sistemas de } \\
\text { controle informatizados } \\
\text { para operação e gestão. } \\
\text { Elabora e aplica } \\
\text { pesquisa de satisfação } \\
\text { pós-venda. } \\
\text { Participa de reuniões } \\
\text { formais com } \\
\text { fornecedores para } \\
\text { avaliar qualidade do } \\
\text { produto. } \\
\text { Possui certificação } \\
\text { nacional (p. ex.: MTur, } \\
\text { ABAV, SINDETUR etc.). } \\
\text { Possui política de } \\
\text { participação nos lucros. } \\
\text { Realiza aavaliação de * Jar } \\
\text { desempenho com. } \\
\text { critérios e regras claras } \\
\text { para todos os }\end{array}$ & $\begin{array}{l}\text { Monitora e utiliza } \\
\text { redes sociais } \\
\text { para promoção e } \\
\text { divulgação. } \\
\text { Participa de } \\
\text { campanhas } \\
\text { publicitárias em } \\
\text { parceria com } \\
\text { outros atores do } \\
\text { setor turístico. }\end{array}$ \\
\hline
\end{tabular}




\begin{tabular}{|c|c|c|c|c|}
\hline \multirow{2}{*}{$\begin{array}{c}\text { NÍVEL DE } \\
\text { ACUMULAÇÃO } \\
\text { DE } \\
\text { COMPETÊNCIAS } \\
\text { TECNOLÓGICAS }\end{array}$} & \multicolumn{4}{|c|}{$\begin{array}{c}\text { MATRIZ DE COMPETÊNCIAS TECNOLÓGICAS PARA O SETOR DE AGENCIAMENTO } \\
\text { DE VIAGENS E OPERAÇÕES TURÍSTICAS }\end{array}$} \\
\hline & $\begin{array}{l}\text { PRODUTOS E } \\
\text { SERVIÇOS }\end{array}$ & $\begin{array}{c}\text { GESTÃO } \\
\text { FINANCEIRA E } \\
\text { DE } \\
\text { INVESTIMENTOS }\end{array}$ & $\begin{array}{l}\text { PLANEJAMENTO E } \\
\text { GESTÃO } \\
\text { (Planejamento, } \\
\text { Qualidade } \\
\text { Atendimento, } \\
\text { Recursos Humanos, } \\
\text { Tecnologia da } \\
\text { Informação) }\end{array}$ & $\begin{array}{l}\text { PROMOÇÃO E } \\
\text { VENDAS }\end{array}$ \\
\hline $\begin{array}{c}\text { (4) } \\
\text { Avançado }\end{array}$ & $\begin{array}{l}\text { Implanta melhorias } \\
\text { contínuas em produtos e } \\
\text { serviços, por meio de } \\
\text { negociação com } \\
\text { fornecedores/prestadores } \\
\text { de serviços. } \\
\text { Estabelece parceria com } \\
\text { outros agentes e/ou } \\
\text { operadores de viagens } \\
\text { para comercialização de } \\
\text { produtos. Oferece } \\
\text { atendimento no local do } \\
\text { potencial cliente. } \\
\text { Elabora e comercializa } \\
\text { pacotes turísticos } \\
\text { customizados. } \\
\text { Planeja a inclusão de } \\
\text { detalhes personalizados } \\
\text { nos produtos e/ou } \\
\text { serviços comercializados } \\
\text { (p. ex.: ingressos para } \\
\text { shows, flores para casal } \\
\text { etc.). } \\
\text { Busca contínua por novos } \\
\text { produtos e serviços para } \\
\text { seus clientes. }\end{array}$ & $\begin{array}{l}\text { Utiliza análise de } \\
\text { retorno de } \\
\text { investimentos } \\
\text { (ROI). } \\
\text { Acompanha } \\
\text { formalmente a } \\
\text { sustentabilidade } \\
\text { financeira. Acessa } \\
\text { fontes de } \\
\text { financiamento de } \\
\text { bancos e } \\
\text { investidores } \\
\text { nacionais e/ou } \\
\text { internacionais. }\end{array}$ & $\begin{array}{l}\text { Projeta tendências } \\
\text { mercadológicas para } \\
\text { segmentos específicos } \\
\text { de mercado. Analisa o } \\
\text { desempenho com base } \\
\text { em metas e indicadores } \\
\text { definidos no plano } \\
\text { estratégico. } \\
\text { Monitora o perfil de } \\
\text { consumo de produtos } \\
\text { turísticos do seu cliente. } \\
\text { Possui um plano de } \\
\text { internacionalização por } \\
\text { meio da compra, venda } \\
\text { ou distribuição de } \\
\text { produtos e/ou serviços } \\
\text { turísticos. } \\
\text { Oferece o serviço } \\
\text { remunerado de } \\
\text { consultoria em viagens. } \\
\text { Compartilha boas } \\
\text { práticas de qualidade } \\
\text { no atendimento com } \\
\text { parceiros fornecedores. } \\
\text { Desenvolve e implanta } \\
\text { plano de } \\
\text { desenvolvimento } \\
\text { profissional do quadro } \\
\text { de funcionários (p.ex.: } \\
\text { plano de carreira). } \\
\text { Realiza mapeapeamento } \\
\text { competede } \\
\text { profissionais com base } \\
\text { nas necessidades } \\
\text { planejadas do negócio. }\end{array}$ & $\begin{array}{l}\text { Participa como } \\
\text { expositor em } \\
\text { feiras e/ou } \\
\text { eventos } \\
\text { internacionais } \\
\text { com foco em } \\
\text { promoção e } \\
\text { divulgação (p.ex.: } \\
\text { Salão do Turismo } \\
\text { de Madri). } \\
\text { Planeja e executa } \\
\text { promoção para o } \\
\text { seu público-alvo } \\
\text { com base em } \\
\text { plano de } \\
\text { marketing } \\
\text { estabelecido. }\end{array}$ \\
\hline
\end{tabular}


Fonte: Adaptado de Figueiredo (2009) e Figueiredo, Gomes e Farias (2010).

\section{Processos Subjacentes de Aprendizado}

De uma forma geral, Bell (1984, 2006), Figueiredo (2003, 2004 e 2009) e Dutrénit (2004) concordam que empresas constroem competências tecnológicas por meio de processos de aprendizagem.

Segundo Bell (1984), apesar do termo 'processo de aprendizagem' ser utilizado com diferentes significados no campo do conhecimento organizacional; pode-se afirmar que o termo aprendizagem refere-se a alguns modos pelos quais uma empresa aumenta sua capacidade para gerenciar e implantar mudanças. Lazonick (2005) corrobora esta argumentação, e acrescenta que o processo de inovação é coletivo e a aprendizagem não pode ser realizada sozinha, a aprendizagem requer a colaboração de pessoas diferentes com capacidades diferentes.

Para Bell (1984), existem dois grandes grupos de mecanismos de aprendizagem. O primeiro grupo de mecanismos, em que o custo é menor para o desenvolvimento da capacitação tecnológica, é representado por: learning-by-operating (aprendendo pela operação) e learning-by-changing (aprendendo pela mudança). E o segundo grupo de mecanismos, em que o processo de aprendizagem depende da alocação de recursos, consiste em: system learning/performance (aprendendo pelo sistema ou desempenho), learning-by-training (aprendendo pelo treinamento), by hiring (aprendendo pela contratação) e by searching (aprendendo pela busca). Outro mecanismo, learning by interacting (aprendizado pela interação) com clientes e fornecedores, também é citado na literatura sobre processos de aprendizagem tecnológicos (p. ex. Lundvall, 1992; Freeman, 1994).

Para Dodgson (1993) e Dodgson et al (2008), aprendizagem é um conceito dinâmico, e sua utilização na teoria enfatiza a mudança contínua na natureza das organizações. Aprendizagem pode ser descrita pelas formas que as empresas constroem e organizam conhecimento e rotinas em torno das atividades, e adaptam e desenvolvem a eficiência organizacional pela melhoria do uso das habilidades de seus funcionários.

Kim (1997) e Kim e Nelson (2000) examinaram, a partir dos estudos de casos da indústria asiática, as trajetórias exitosas de acumulação de competências tecnológicas e a importância dos processos de conversão de aprendizagem individual para aprendizagem organizacional, associados a essas trajetórias. Dutrénit (2000), baseada em estudo de caso de uma indústria de vidro no México, focalizou as limitações em criar uma base de conhecimento coerente para desenvolver competências tecnológicas no longo prazo, e verificou que o processo de aprendizagem irregular da empresa sofreu influência de diversos fatores, sendo os seguintes fatores centrais: os limitados esforços para converter conhecimento do nível individual para o nível organizacional; as diferentes estratégias de aprendizagem buscadas pela empresa e as limitações em termos de coordenação; e a instabilidade do processo de criação de conhecimento. A autora argumentou que os processos de aprendizagem intraorganizacionais desempenharam papel importante, influenciando a trajetória de acumulação de competências tecnológicas da empresa.

Apesar das diversas pesquisas realizadas, na literatura de empresas em industrialização, estes estudos ainda apresentam limitações para explicar como os processos de aprendizagem influenciam a acumulação de competências tecnológicas em empresas e como essas questões diferem entre empresas. Além disso, poucos são os estudos que tratam de pequenas e médias empresas, sobretudo no setor de Turismo Brasil (Cavalcanti \& Figueiredo, 2006; Figueiredo et al 2010). Ademais a própria literatura sobre operadores e agências de viagens no Brasil é escassa, como aponta Trigo \& Arendit (2007, pp. 329).

Estrutura Analítica - Processos de Aprendizagem

Com base na literatura, a estrutura analítica de aprendizagem foi decomposta em dois processos distintos: 1) processos de aquisição de conhecimento e 2) processos de conversão de conhecimento (Nonaka \& Takeuhci, 


\section{Trajetória de Acumulação de Competências Tecnológicas e Processos de Aprendizagem: proposta de modelo analítico para agências de viagens e operadoras turísticas}

1997; Figueiredo, 2009; Figueiredo et al, 2010), sendo processos de aquisição de conhecimento mais diretamente relacionados com o nível individual de aprendizagem, e o segundo, processos de conversão, com 0 nível organizacional.

Processos de aquisição de conhecimento têm sido tratados tanto na literatura de empresas de países em industrialização (p. ex.: Katz, 1984, 1997, 2001; Lall, 1987, 1992; Hobday, 1995; Kim, 1997, 1999; Kim et al, 1987; Kim \& Nelson, 2000; Dutrénit, 2004, 2005; Figueiredo, 2001, 2009) como na literatura de empresas de tecnologia de fronteira (p. ex.: Cohen \& Levinthal, 1989, 1990; Leonard-Barton, 1998; Bell \& Pavitt, 1993; Cantwell, 1989; Maskell \& Malmberg, 1999). A estrutura proposta a seguir se baseia em Figueiredo (2002a, 2009, 2010) e foi elaborada com base nas perspectivas de 'aprendizagem organizacional' (Cyert \& March, 1963; Hedberg, 1981; Argyris \& Schön, 1978; Dodgson, 1993; Bessant, 1998; entre outros) e da 'firma geradora de conhecimento' (Nelson \& Winter, 1982; Winter, 1988; Teece et al., 1988; Nonaka \& Takeuchi, 1997; LeonardBarton, 1998; Leonard \& Sensiper, 1998).

A Tabela 2 apresenta a estrutura para processos de aprendizagem. As linhas dispõem os quatro processos de aprendizagem; as colunas e as quatro características chave desses processos.

i) Processos de aquisição externa de conhecimento - são os processos pelos quais os indivíduos adquirem conhecimento tácito e/ou explícito do ambiente externo à empresa. Por exemplo: importação de expertise ou especialistas de fora da empresa, treinamento no exterior, participação em conferências e eventos relacionais, provisão de bolsas de estudo, interações com fornecedores e usuários; entre outros.

ii) Processos de aquisição interna de conhecimento - são os processos pelos quais indivíduos adquirem conhecimento tácito e/ou codificado dentro da empresa. Por exemplo: atividades on-the-job/learning-byoperating (aprender-fazendo) como operação de rotina da planta, envolvimento em desenho de projetos, em projetos de instalação, estudos sistemáticos em laboratórios, aprimoramento contínuo das plantas; entre outros.

iii) Processos de socialização de conhecimento - são os processos pelos quais indivíduos partilham seu conhecimento tácito. Ou seja, quaisquer processos formais ou informais pelos quais conhecimento tácito é transferido de um indivíduo ou grupo para outro.Por exemplo: treinamentos internos básicos ou avançado, construção de times, disseminação de operadores líderes, solução compartilhada de problemas, entre outros.

iv) Processos de codificação de conhecimento - são os processos pelos quais o conhecimento tácito dos indivíduos, ou parte dele, torna-se explícito. Este conhecimento explícito é articulado em formato organizado e acessível, tornando-se fácil seu entendimento. Por exemplo: práticas de padronização, procedimentos de produção, sistemas de automação, descrição de treinamento externo, manuais de sistemas de qualidade, entre outros.

TABELA 2 - Processos de aprendizagem

Características Chave dos Processos de Aprendizagem 
Saulo Barroso Rocha e Deborah Moraes Zouain

\begin{tabular}{|c|c|c|c|c|}
\hline $\begin{array}{l}\text { PROCESSOS DE } \\
\text { APRENDIZAGEM }\end{array}$ & Variedade & Intensidade & Funcionamento & Interação \\
\hline & $\begin{array}{l}\text { Ausente - Presente } \\
\text { [Limitada - Moderada } \\
\text { - Diversa] }\end{array}$ & $\begin{array}{c}\text { Uma vez - Intermitente } \\
\text { - Contínuo }\end{array}$ & Ruim - Moderado - Bom & $\begin{array}{c}\text { Fraca - Moderada - } \\
\text { Forte }\end{array}$ \\
\hline & Processos e & anismos de aquisiçãc & do conhecimento & \\
\hline $\begin{array}{c}\text { AQUISIÇÃO } \\
\text { EXTERNA DE } \\
\text { CONHECIMENTO }\end{array}$ & $\begin{array}{l}\text { Presença/ausência de } \\
\text { processos para adquirir } \\
\text { conhecimento no Brasil } \\
\text { e/ou no exterior. }\end{array}$ & $\begin{array}{l}\text { O modo como a } \\
\text { empresa usa este } \\
\text { processo ao longo do } \\
\text { tempo pode ser } \\
\text { contínuo, intermitente } \\
\text { ou ocorrer apenas uma } \\
\text { vez. }\end{array}$ & $\begin{array}{l}\text { O modo como o processo } \\
\text { é criado (p.ex.: critério } \\
\text { para envio de funcionários } \\
\text { para treinamento externo) } \\
\text { e o modo como ele opera } \\
\text { ao longo do tempo podem } \\
\text { fortalecer ou mitigar } \\
\text { variedade e intensidade. }\end{array}$ & $\begin{array}{l}\text { O modo como um } \\
\text { processo influencia } \\
\text { outro processo de } \\
\text { aquisição externa ou } \\
\text { interna de } \\
\text { conhecimento e/ou } \\
\text { outros processos de } \\
\text { conversão do } \\
\text { conhecimento. }\end{array}$ \\
\hline $\begin{array}{c}\text { AQUISIÇÃO } \\
\text { INTERNA DE } \\
\text { CONHECIMENTO }\end{array}$ & $\begin{array}{l}\text { Presença/ausência de } \\
\text { processos para adquirir } \\
\text { conhecimento } \\
\text { realizando atividades } \\
\text { internas. }\end{array}$ & $\begin{array}{l}\text { O modo como a } \\
\text { empresa usa diferentes } \\
\text { processos para } \\
\text { aquisição interna de } \\
\text { conhecimento pode } \\
\text { influenciar o } \\
\text { entendimento pelos } \\
\text { indivíduos dos princípios } \\
\text { envolvidos na } \\
\text { tecnologia. }\end{array}$ & $\begin{array}{l}\text { O modo como o processo } \\
\text { é criado e o modo como } \\
\text { ele opera ao longo do } \\
\text { tempo tem implicações } \\
\text { práticas para variedade e } \\
\text { intensidade. }\end{array}$ & $\begin{array}{l}\text { Processos de } \\
\text { aquisição interna } \\
\text { podem ser } \\
\text { influenciados por } \\
\text { processos de } \\
\text { aquisição externa. } \\
\text { Isso pode influenciar } \\
\text { processos de } \\
\text { conversão de } \\
\text { conhecimento. }\end{array}$ \\
\hline & Processos & ecanismos de convers & o de conhecimento & \\
\hline $\begin{array}{c}\text { SOCIALIZAÇÃO } \\
\text { DE } \\
\text { CONHECIMENTO }\end{array}$ & $\begin{array}{l}\text { Presença/ausência de } \\
\text { diferentes processos } \\
\text { pelos quais indivíduos } \\
\text { compartilham seu } \\
\text { conhecimento tácito. }\end{array}$ & $\begin{array}{l}\text { O modo como os } \\
\text { processos prosseguem } \\
\text { ao longo dos anos. } \\
\text { Intensidade contínua } \\
\text { dos processos de } \\
\text { socialização pode } \\
\text { influenciar codificação } \\
\text { de conhecimento. }\end{array}$ & $\begin{array}{l}\text { O modo como } \\
\text { mecanismos de } \\
\text { socialização de } \\
\text { conhecimento são criados } \\
\text { e operam ao longo do } \\
\text { tempo. Isso tem } \\
\text { implicações para a } \\
\text { variedade e intensidade do } \\
\text { processo de conversão de } \\
\text { conhecimento. }\end{array}$ & $\begin{array}{l}\text { Condução de } \\
\text { diferentes } \\
\text { conhecimentos } \\
\text { tácitos para um } \\
\text { sistema efetivo (p. } \\
\text { ex.: criação de 'links' } \\
\text { de conhecimento). } \\
\text { Socialização pode } \\
\text { ser influenciada por } \\
\text { processos de } \\
\text { aquisição externa e } \\
\text { interna de } \\
\text { conhecimento. }\end{array}$ \\
\hline
\end{tabular}




\section{Trajetória de Acumulação de Competências Tecnológicas e Processos de Aprendizagem: proposta de modelo analítico para agências de viagens e operadoras turísticas}

\begin{tabular}{|c|c|c|c|c|}
\hline $\begin{array}{c}\text { CODIFICAÇÃO } \\
\text { DE } \\
\text { CONHECIMENTO }\end{array}$ & $\begin{array}{l}\text { Presença/ausência de } \\
\text { diferentes processos e } \\
\text { mecanismos para } \\
\text { codificar o } \\
\text { conhecimento tácito. }\end{array}$ & $\begin{array}{l}\text { O modo como } \\
\text { processos de } \\
\text { padronização são } \\
\text { repetidamente } \\
\text { realizados. Codificação } \\
\text { ausente e/ou } \\
\text { intermitente pode limitar } \\
\text { a aprendizagem } \\
\text { organizacional. }\end{array}$ & $\begin{array}{l}\text { O modo como a } \\
\text { codificação de } \\
\text { conhecimento é criada e } \\
\text { opera ao longo do tempo } \\
\text { tem implicações para o } \\
\text { funcionamento de todo o } \\
\text { processo de conversão de } \\
\text { conhecimento. Isso } \\
\text { também influencia } \\
\text { variedade e intensidade do } \\
\text { processo. }\end{array}$ & $\begin{array}{l}\text { O modo como } \\
\text { codificação de } \\
\text { conhecimento é } \\
\text { influenciada por } \\
\text { processos de } \\
\text { aquisição de } \\
\text { conhecimento ou } \\
\text { por processos de } \\
\text { socialização de } \\
\text { conhecimento (p. } \\
\text { ex.: construção de } \\
\text { times). }\end{array}$ \\
\hline
\end{tabular}

Fonte: Figueiredo (2002, 2009, 2010), com base em Cyert e March (1963); Hedberg (1981); Argyris e Schön (1978); Dodgson (1993); Bessant (1998); Nonaka e Takeuchi (1997); Leonard-barton (1998); e Leonard e Sensiper (1998)

As características chave dos processos de aprendizagem são: variedade, intensidade, funcionamento e interação, definidas abaixo:

i) Variedade - consiste na presença de diferentes processos de aprendizagem dentro da empresa. É avaliada em termos de presença/ausência de um processo inteiro (p. ex.: processo de aquisição interna de conhecimento) e os subprocessos que este pode conter (p. ex.: operação de rotina de um Sistema Global de Distribuição). A Variedade é avaliada em todos os processos e dentro destes.

ii) Intensidade - refere-se à recorrência no decurso da criação, atualização, uso, aprimoramento e/ou fortalecimento dos processos de aprendizagem. Intensidade é importante porque i) pode assegurar um fluxo constante de conhecimento externo para a empresa; ii) pode levar a um maior entendimento de novos produtos ou processos; e iii) pode assegurar uma conversão constante de aprendizagem individual para o nível organizacional. Esta característica apresenta-se sob três aspectos: uma vez, intermitente e contínua.

iv) Funcionamento - é o modo pelo qual empresas organizam seus processos de aprendizagem, é crítico para a construção de competências. 'Funcionamento' é definido como o modo pelo qual os processos de aprendizagem operam ao longo do tempo. Embora a intensidade possa ser contínua, o funcionamento dos processos pode ser insuficiente (repetição de erros). 0 funcionamento pode contribuir para fortalecer e/ou mitigar as características variedade e intensidade. Esta característica apresenta-se sob três aspectos: fraco, moderado, bom.

v) Interação - é o modo pelo qual os processos de aprendizagem influenciam um ao outro. A interação entre os processos de aquisição e conversão de conhecimento é importante para a construção de competências. Esta característica apresenta-se sob três aspectos: fraca, moderada ou forte.

As estruturas analíticas para acumulação de competências tecnológicas e processos de aprendizagem servem de base para descrever e analisar as evidências em empresas do setor de agenciamento de viagens e operações turísticas. À luz dessas estruturas, pode-se identificar o nível de competência tecnológica acumulada em determinada empresa do setor de agenciamento de viagens e operações turísticas, bem como a relação 
entre processos de aprendizagem sobre a construção da trajetória de acumulação destas competências ao longo do tempo.

\section{Comentários Finais}

Existe um consenso na literatura sobre inovação que empresas constróem competências ou capacidades tecnológicas por meio de processos de aprendizagem. Assim, a aprendizagem tecnológica se refere ao processo dinâmico de aquisição daquelas capacidades, básicas ou inovadoras. Normalmente, este processo ocorre com base em escolhas sob o contexto de um paradigma econômico e técnico (Bell, 1984, 2006; Bell \& Pavitt, 1995; Bell \& Giuliani, 2007; Bessant, 1998; Figueiredo, 2002; Nelson \& Winter, 1977, 1982; Dosi, 1988).

Com base em modelos analíticos desenvolvidos para avaliar capacidades inovadoras e tecnológicas em empresas, notadamente, do setor industrial (Archibugi, 2001; Ariffin \& Figueiredo, 2004; Bell, 1984; Bell \& Pavitt, 1993; Bell \& Giuliani, 2007; Cantwell, 1989; Cohen \& Levinthal, 1989; Dutrénit \& Vera-Cruz, 2005; Figueiredo, 2002, 2003; Karaoz \& Albeni, 2005; Katz, 1987; Kim, 1997), esta pesquisa propõe uma estrutura de análise (Tabela 1 - Matriz de Competências Tecnológicas) para empresas do setor de agenciamento de viagens e operações turísticas. A aplicabilidade do modelo, concebido inicialmente no contexto de organizações industriais para o setor de serviços turísticos, alinha-se com a proposta de Castellacci (2008), De Jong e Marsili (2006), Guerrieri e Meliciani (2005), para quem existem mais pontos em comum do que divergentes nas diferentes taxonomias utilizadas nos dois setores. Ressalta-se que na ampla literatura mencionada, sobre aprendizagem tecnológica para acumulação de capacidades ou competências tecnológicas e inovadoras, são poucas as referências a estudos desta natureza em empresas puramente de serviços (Cavalcanti \& Figueiredo, 2006; Figueiredo et al, 2010), como agentes de viagens e operadoras de turismo, que de fato atuem como distribuidores de produtos, de interconexão entre oferta e demanda.

Para o setor de agenciamento de viagens e de operação turística, ocorreu a mudança de um importante paradigma técnico-econômico durante os anos 90 e principalmente na recém terminada década de 2000 . No contexto de fatores a constar de um novo paradigma, foram definidas competências tecnológicas, como: incorporação de novas tecnologias de informação às operações, novos produtos e serviços para clientes. Fatores observados no ambiente externo às agências e operadoras também tem influenciado diretamente 0 desempenho deste setor. Dentre algumas fatores externos, citam-se: macroeconômico, político, ambientais e demográficos (Dwyer et al., 2009). Estas variáveis do ambiente externo brasileiro impõem a mesma implicação gerenciais para todos as agências de viagens. A capacidade de entender estas implicações e a habilidade de transformá-las em oportunidades esta relacionada as competências tecnológicas instaladas nas empresas brasileiras do setor. A diferenciação se dará também pelo melhor aproveitamento dos processos de aprendizagem para construir as competências tecnológicas necessárias para atuar neste mercado.

Portanto, a generalização analítica do modelo aqui proposto, ou seja, da aplicação das estruturas desenvolvidas para agências e operadoras é uma possibilidade real para o desenvolvimento de competências tecnológicas no setor de agenciamento de viagens e operações turísticas, entendendo como os diversos processos de aprendizagem influenciam na trajetória de acumulação destas competências nas organizações.

\section{Referências}

Abernathy, W. \& Clark, K. (1985) "Innovation: mapping the winds of creative destruction". Research Policy, v.14, p.3-22.

Alexander, J. \& Carayannis, E. G. (2002) "Is technological learning a firm core competence, when, how and why? A longitudinal, multi-industry study of firm technological learning and market performance". Technovation, v. 22, p. 625-643. 


\section{Trajetória de Acumulação de Competências Tecnológicas e Processos de Aprendizagem: proposta de modelo analítico para agências de viagens e operadoras turísticas}

Archibugi, D. (1990) "Sources of innovative activities and industrial organization in Italy". Research Policy, v. 20, p. 299-313.

Argyris, C. \& Schön, D. (1978) "Organization learning: A theory of Action perspective". Reading, Mass: Addison Wesley.

Ariffin, N. \& Bell, M. (1999) "Firms, politics and political economy: patterns of subsidiary-parent linkages and technological capability-building in electronics TNC subsidiaries in Malaysia". In: JOMO, K. S.; RASIAH, R.; FELKER, G. (Eds.). Industrial technology development in Malaysia. London: Routledge.

Ariffin, N. \& Figueiredo, P. N. (2004) "Internalisation of innovative capabilities: counter-evidence from the electronics industry in Malaysia and Brazil". Oxford Development Studies, v. 32, p. 559-583.

Arrow, K. (1962) "The economic implications of learning by doing". Economic Studies, v. 29, p. 155-173.

Bell, M. (1984) "Learning and the accumulation of industrial technological capacity in developing countries". In: King, K. \& Fransman, M. (eds.). Technological capability in the Third World. London: Macmillan.

. (2006) "Time and technological learning in industrializing countries: how long does it take? How fast is it moving (if at all)?" International Journal of Technology Management, v. 36, p. 25-42.

Bell, M. \& PAVITT, K. (1993) "Technological accumulation and industrial growth: contrasts between developed and developing countries". Industrial and Corporate Change. London, v. 2, n. 2, p. 157-211.

(1995) "The development of technological capabilities". In: UL HAQUE, I. (ed.). Trade, Technology and International Competitiveness. Washington, DC: The World Bank.

Bell, Martin, Ross-Larsen, B. \& Westphal, L. (1984) "Assessing the performance of infant industries". Journal of Development Economics, v. 16, p. 101-128.

Bell, M. \& Giuliani, E. (2007) "Catching up in the global wine industry: innovation systems, cluster knowledge networks and firm-level capabilities in Italy and Chile". International Journal of Technology and Globalisation, v. 3,p. 197-223.

Bessant, J. (1998) "Developing continuous improvement capability". International Journal of Innovation Management, v. 2, p. 409-429.

BRASIL. Ministério do Turismo. (2008a) "Estudo da demanda turística internacional 2005-2007". Brasília, DF.

Ministério do Turismo. (2008b) "Pesquisa anual de conjuntura econômica do Turismo 2008". Brasília,

DF.

\section{1.}

Ministério do Turismo. (2011) "Pesquisa anual de conjuntura econômica do Turismo 2008". Brasília, DF,

Bruland, K. \& Mowery, D. C. (1989) "Innovation through time". In: FAGERBERG, Jan, CANTWELL, John. Technological innovation and multinational corporations. Blackwell: Oxford.

Castellacci, F. (2008) "Technological paradigms, regimes and trajectories: manufacturing and service industries in a new taxonomy of sectoral patters of innovation". Research Policy, v. 37, p. 978-994.

Cavalcanti, G. \& Figueiredo, P. N. (2006) "Voando alto e baixo: competências técnico-organizacionais e performance competitiva em microempresas de turismo de aventura no Rio de Janeiro". Revista do Observatório de Inovação do Turismo, v. 1, n.2.

Cohen, W. M. \& Levinthal, D. A. (1989) Innovation and learning: the two faces of R \& D. Economic Journal, v. 99, p. 569-596.

(1990) Absorptive capacity: a new perspective on learning and innovation. Administrative Science Quaterly, v. 35, p. 123-138.

Cyert, R. \& March, J. (1963) "Behavioral Theory of the Firm". Oxford: Blackwell. 
Dahlman, C., Ross-Larsen, B. \& Westphal, L. E. (1987) "Managing technological development: lessons from newly industrializing countries". World Development, v.15, p.759-775.

De Jong, J. P. \& Marsili, O. (2006) "The fruit flies of innovations: a taxonomy of innovative small firms". Research Policy, v. 35, p. 213-229.

Dodgson, M. (1993) "Organisational learning: a review of some literatures". Organisation Studies. Brighton, v. 14, n. 3, p. 375-394.

Dodgson, M., Gann, D. \& Salter, A. (2008) "The management of technological innovation". London: Oxford University Press, 2008.

Dosi, G., Pavitt, K. \& Soete, L. (1992) "The Economics of Technical Change and. International Trade". London:: Harvester-Wheatsheaf.

Dosi, G. (1982) "Technical paradigms and technical trajectories, the determinants and directions of technical change and the transformation of the economy". Research Policy, v.11, p. 147-162.

(1988a) "The nature of the innovative process". In: DOSI, Giovanni et al. (org.). Technical Change and Economic Theory. London: Pinter Publishers.

(1988b) "Sources, procedures, and microeconomic effects of innovation". Journal of Economic Literature. London, Vol. XXVI, p. 1120-1171, 1988b.

(1997) "Opportunities, incentives and collective patterns of technological change". The Economic Journal. V. 107, n. 444, p. 1530-1547.

Dosi, G. \& Orsenigo, L. (1988) "Coordination and transformation: an overview of structures, behaviours and change in evolutionary environments". In: DOSI, Giovanni et al. (org.). Technical Change and Economic Theory. London: Pinter Publishers.

Dosi, G., Marengo, L. \& Pasquali, C. (2006) "How much should society fuel the greed of innovators? On the relations between appropriability, opportunities and rates of innovation". Research Policy, v. 35, p. 110-1121.

Dutrénit, G. (2000) "Learning and Knowledge Management in the Firm: From Knowledge Accumulation to Strategic Capability". Cheltenham, UK, Northhamptom, MA, USA, Edward Elgar.

(2004) "Building technological capabilities in latecomer firms: a review essay". Science Technology Society, v.9, p.209-241.

DutrénitT, G. \& Vera-Cruz, A. O. (2005) "Technological capability accumulation in the maquila industry in Mexico". Cadernos EBAPE, Edição Especial. Disponível em www.ebape.fgv.br/cadernosebape.

Dwyer, L. Edwards, D. Mistilis, N., Roman, C. Scott, N. (2009) "Destination and enterprise management for a tourism future". Tourism Management, v. 30, p. 63-74

Evangelista, R. (2000) "Sectoral patterns of technological change in services". Economics of Innovation and New Technology, v. 9, p. 183-221.

Figueiredo, P. N. (2000) "Trajetórias de acumulação de competências tecnológicas e os processos subjacentes de aprendizagem: revisando estudos empíricos". Revista de Administração Pública. Rio de Janeiro, v. 34, n. 1, p. 7-33, Jan./Fev.

. (2001) "Technological learning and competitive performance". Cheltenham: Edward Elgar.

(2002a) "Does technological learning pay off? Inter-firm differences in Technological capabilityaccumulation paths and operational performance improvement". Research Policy, v. 31, p.73-94.

(2003) "Learning, capability-accumulation and firms difference: evidence from latecomer steel". Industrial and Corporate Change, v. 12, p.607-643.

. (2004) "Aprendizagem tecnológica e inovação industrial: conceitos, mensuração e evidências do Brasil".

Revista Brasileira de Inovação, v. 3, jul/dez.

LTC.

. (2009) "Gestão da inovação: conceitos, métricas e experiências de empresas no Brasil". Rio de Janeiro:

Figueiredo, P., Gomes, S. \& Farias, R. (2010) "Innovative technological capability in firms of the tourism sector: a study of the hotels in the city of Rio de Janeiro during the 1990-2008 periodo". Revista de Administração Pública, v. 44, set/out, p. 1139-1170. 


\section{Trajetória de Acumulação de Competências Tecnológicas e Processos de Aprendizagem: proposta de modelo analítico para agências de viagens e operadoras turísticas}

Freeman, C. (1982) "The economics of industrial innovation". 2nd edition. London: Pinter. (1988) "Introduction". In: DOSI, G. et al (org.). "Technical Change and Economic Theory". London: Pinter Publishers.

"The economics of technical change: a critical survey". Cambridge Journal of Economics, v. 18, 1994.

Gallouj, F. \& Olivier, W. (1997a) "Innovation in services". Research Policy, v. 26, p. 537-556, 1997.

(1997b) "Innovation theory: towards a neo-Schumpeterian theory of innovation in services?" Research

Policy, v. 24, n. 6, p. 405-420.

Hedberg, B. (1981) "How organizations learn and unlearn". In: NYSTROM, P. C.,

Hobday, M. (1995) "Innovation in East Asia: the challenge to Japan". Aldershot: Edward Algar.

Karaoz, M., Albeni, M. (2005) "Dynamic technological learning trends in Turkish manufacturing industries". Technological Forecasting \& Social Change, v. 72, p. 866-885.

Katz, J. (1984) "Domestic technological innovation and dynamic comparative advantage". Journal of Development Economics, v. 16, p.13-37.

. (1987) "Technology generation in Latin America manufacturing industries". London: Macmillan.

(1997) "New problems and opportunities for industrial development in Latin America". Oxford Development Studies, v.25, n. 3.

(2001) "Structural reforms and technological behavior: the sources and nature of technological change in Latin America in the 1990s". Research Policy, v. 30, p. 1-19.

Kim, L. (1997) "The Dynamics of Samsung's Technological Learning in Semiconductors". California Management Review, vol 39, no. 3, p. 86-100.

- (1999) Learning and innovation in economic development. Cheltenham: Edward Elgar.

Kim, L. \& Nelson, R. (2000) Technology, Learning and Innovation: the experience of the Asian NIEs. Cambridge: Cambridge University Press.

Lall, S. (1987) "Learning to industrialize: the acquisition of technological capability by India". London: Macmillan Press.

186.

(1992) "Technological capabilities and industrialization". World Development. London, v. 20, n. 2, p. 165-

Lam, A. (2005) Organizational innovation. In: FAGERBERG, Jan, MOWERY, David C., NELSON, Richard R (Orgs.). The Oxford Handbook of Innovation. New York, Oxford University Press.

Lazonick, W. (2005) "The innovative firm". In: FAGERBERG, Jan, MOWERY, David C., Nelson, R. (Orgs.). The Oxford Handbook of Innovation. New York, Oxford University Press.

Leonard-Barton, D. (1998) "Nascentes do Saber: criando e sustentando as fontes de inovação". Rio de Janeiro: Fundação Getulio Vargas.

Leonard, D. \& Sensiper, S. (1998) "The role of tacit knowledge in group innovation". California Management Review, v. 40, p.112-125.

Lundvall, B. A. (Ed.). (1992) "National Systems of Innovation: towards a theory of innovation and interaction learning". London: Pinter.

Maskell, P. \& Malmberg, A. (1999) "Localised learning and industrial competitiveness". Cambridge Journal of Economics, v. 23, p. 167-186.

Miles, I. (2005) "Innovation in services". In: FAGERBERG, Jan, MOWERY, David C., NELSON, Richard R (Orgs.). The Oxford Handbook of Innovation. New York, Oxford University Press.

Nonaka, I. \& Takeuchi, H. (1997) "Criação de Conhecimento na Empresa: como as empresas japonesas geram a dinâmica da inovação". Rio de Janeiro: Campus, 1997. 
O'Sullivan, M. (2000) The innovative enterprise and corporate governance. Cambridge Journal of Economics, v. 24, p.393-416.

Organização Mundial do Turismo. (2010) Barômetro Mundial do Turismo. Vol. 8 Número 1. OMT.

Patel, P. \& Pavitt, K. (1992) "The innovative performance of the world's largest firms: some new evidence". The economics of innovation and new technology, v. 2, p. 91-102.

"Uneven (and divergent) technological accumulation among advanced countries: evidence and a framework of explanation". Industrial and Corporate Change, v. 3, n. 3, 1994.

Pavitt, K. (1984) "Sectoral patterns of technical change: Towards a taxonomy and a theory". Research Policy. Brighton, v. 13, n. 6, p. 343-373, 1984.

(2005) "Innovation processes". In: FAGERBERG, Jan, MOWERY, David C., NELSON, Richard R (Orgs.). The Oxford Handbook of Innovation. New York, Oxford University Press.

Rosenberg, N. (1982) "Inside the blackbox". Cambridge: Cambridge University Press.

Souitaris, V. (2002) Technological trajectories as moderators of firm-level determinants of innovation. Research Policy, v. 31, p. 877-898.

Teece, D. (1977) Technology transfer by multinational firms: the resource cost of transferring technological knowhow. Economic Journal, p. 242-261, Junho.

. (1986) "Profiting from technological innovation". Research Policy, v. 15, p. 285-306.

Teitel, S. (1981) Towards an understanding of technical change in semi-industrialized countries. Research Policy, v. 10, p. 127-147.

Trigo, L.G. \& Arendit, E. J. (2007) "La inestabilidad del Mercado turístico: desde la perspectiva de una agencia de viajes brasileña". Estudios y Perspectivas en Turismo, v. 6, p. 323-340. 